\title{
Computational Theory of Warm Condensed Matter
}

T.W. Barbee, M.P. Surh, L.X. Benedict

February 25, 2001

U.S. Department of Energy

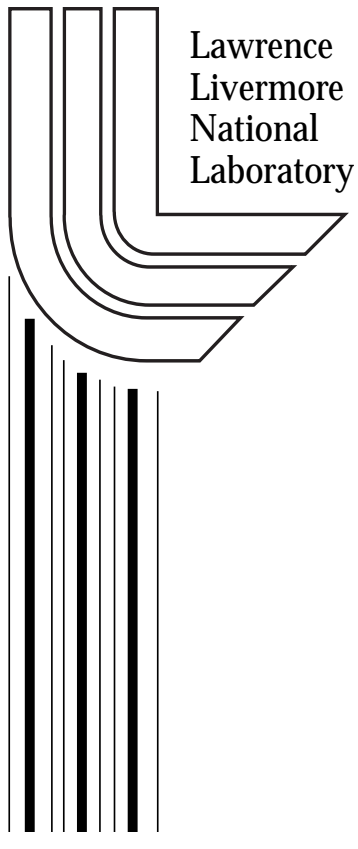




\section{DISCLAIMER}

This document was prepared as an account of work sponsored by an agency of the United States Government. Neither the United States Government nor the University of California nor any of their employees, makes any warranty, express or implied, or assumes any legal liability or responsibility for the accuracy, completeness, or usefulness of any information, apparatus, product, or process disclosed, or represents that its use would not infringe privately owned rights. Reference herein to any specific commercial product, process, or service by trade name, trademark, manufacturer, or otherwise, does not necessarily constitute or imply its endorsement, recommendation, or favoring by the United States Government or the University of California. The views and opinions of authors expressed herein do not necessarily state or reflect those of the United States Government or the University of California, and shall not be used for advertising or product endorsement purposes.

This work was performed under the auspices of the U. S. Department of Energy by the University of California, Lawrence Livermore National Laboratory under Contract No. W-7405-Eng-48.

This report has been reproduced directly from the best available copy.

Available electronically at http://www.doc.gov/bridge

Available for a processing fee to U.S. Department of Energy

And its contractors in paper from

U.S. Department of Energy

Office of Scientific and Technical Information

P.O. Box 62

Oak Ridge, TN 37831-0062

Telephone: (865) 576-8401

Facsimile: (865) 576-5728

E-mail: reports@adonis.osti.gov

Available for the sale to the public from

U.S. Department of Commerce

National Technical Information Service

5285 Port Royal Road

Springfield, VA 22161

Telephone: (800) 553-6847

Facsimile: (703) 605-6900

E-mail: orders@ntis.fedworld.gov

Online ordering: http://www.ntis.gov/ordering.htm

OR

Lawrence Livermore National Laboratory

Technical Information Department's Digital Library

http://www.llnl.gov/tid/Library.html 


\title{
COMPUTATIONAL THEORY OF WARM CONDENSED MATTER
}

\author{
Troy W. Barbee III, Michael P. Surh, and Lorin X. Benedict \\ 98-ERD-052
}

\begin{abstract}
We have developed an improved computational theory of condensed matter in the regime where

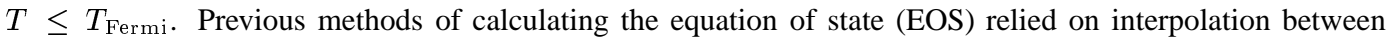
low-temperature (solid) and high-temperature (plasma) limits, or employed severe approximations. Recent theoretical and experimental developments have highlighted the need for accurate EOS and opacity data in the intermediate temperature range and offer the opportunity to test theoretical models. We describe our results for EOS and optical properties for temperatures up to $10^{6} \mathrm{~K}$, and describe directions for future work.
\end{abstract}

\section{INTRODUCTION}

Laboratory missions such as the stockpile stewardship program (SSP) demand accurate materialsmodeling capabilities. Among these, calculations of the equation of state (EOS) and opacity play important roles. SSP programs require knowledge of these properties over an extreme range of densities and temperatures. In the low-density/high-temperature limit, models based on plasma physics are very accurate, whereas models based on condensed-matter physics perform best in the high-density/low-temperature regime. In this project, we are focusing on "warm" condensed matter, i.e., on the region of intermediate temperature and density where the high-temperature or low-temperature approximations may no longer be valid.

Tremendous advances in condensed-matter theory over the past few decades have led to the ability to predict the electronic, vibrational, and superconducting properties of both real and hypothetical materials from "first principles," i.e., using only the atomic number $\mathrm{Z}$ and mass $\mathrm{A}$ as inputs to the calculation. The vast majority of this theoretical effort has been focused on the low temperature limit and relatively little attention has been given to warm, dense states of matter.

Current models for the EOS of warm, dense matter are primarily based on theoretical models that are most accurate in the low-temperature limit and that treat the effect of finite temperature as a perturbation of the zero-temperature solution. These models are largely untested above the melting point-especially approaching the Fermi temperature $T_{\text {Fermi }}$ (the characteristic energy scale for the interacting electrons in a material, typically on the order of a few $10^{5} \mathrm{~K}$ ). Thus, the goal of the current project is to develop rigorous extensions of existing state-of-the-art condensed-matter techniques that are valid in this intermediate-temperature regime. Our approach focuses on three areas: improving theoretical treatments of the thermal contributions to the EOS; calculating optical properties (e.g., opacity) at finite temperature; and developing a new theoretical formalism that can be used to solve the quantum-mechanical, many-electron problem at finite temperature.

\section{RESULTS}

Our starting point is the case of a crystalline solid at zero temperature. An excellent description of the formalism for calculating the properties of solids at zero temperature can be found in the review article by Payne et al. [1]. Important features of their treatment include: (1) the use of crystalline symmetry to reduce the simulation cell size to only a few (perhaps only one) atoms, greatly simplifying the calculation; (2) the use of pseudopotentials to eliminate core electrons that do not participate in chemical bonding; and (3) the use of an exchange-correlation potential to model the many-body quantum-mechanical behavior of the electrons. 
At finite temperature, this model will begin to break down. First, some of the electrons will occupy excited states due to thermal excitations. At higher temperatures, the solid will melt, resulting in a loss of symmetry, and the thermal motion of the nuclei (or ion cores in the pseudopotential model) will become important. At still higher temperatures, the core electrons will become thermally excited, and will have to be included in the calculation. Additionally, the exchange-correlation potentials $V_{x c}$ used in nearly all condensed matter calculations are based on a zero-temperature model of the electron gas and may not be accurate at high temperatures.

At temperatures below and near the melting temperature (on the order of $10^{3} \mathrm{~K}$ for most solids), the motion of the ions is the most significant factor, and quantum molecular dynamics has been extremely successful in describing the properties of condensed matter up to $T \leq 10^{4} \mathrm{~K}$. Above this temperature, excitation of core electrons will occur at a temperature near $10^{\overline{5}} \mathrm{~K}$, although this is strongly dependent on the material. The use of a $T=0$ model for $V_{x c}$ will begin to fail as $T$ approaches $T_{\text {Fermi }}$, also in the range of $10^{5} \mathrm{~K}$.

We began by modifying our existing codes to consider a finite electron temperature. This improvement allowed us to model experiments [2] in which a semiconductor crystal heated by a femtosecond-pulse laser exhibited thermally induced structural instabilities within a few picoseconds $\left(10^{-12} \mathrm{~s}\right)$ of heating. On this short a time scale, the electrons remain hot while the ions are relatively cold; therefore, melting of the crystal cannot explain the experimental results. It has been proposed that heating the electrons into excited states alters the forces that act on the nuclei, resulting in the observed structural instability. To simulate the experimental conditions, we used the codes we developed to calculate the vibrational properties of a silicon crystal with cold (stationary) ions and hot electrons, simulating the experimental conditions. Figure 1 shows that at a sufficiently high electron temperature (a few eV), the crystal lattice becomes unstable-as reflected by a negative force constant. This calculated result agrees with what was seen in the experiment.

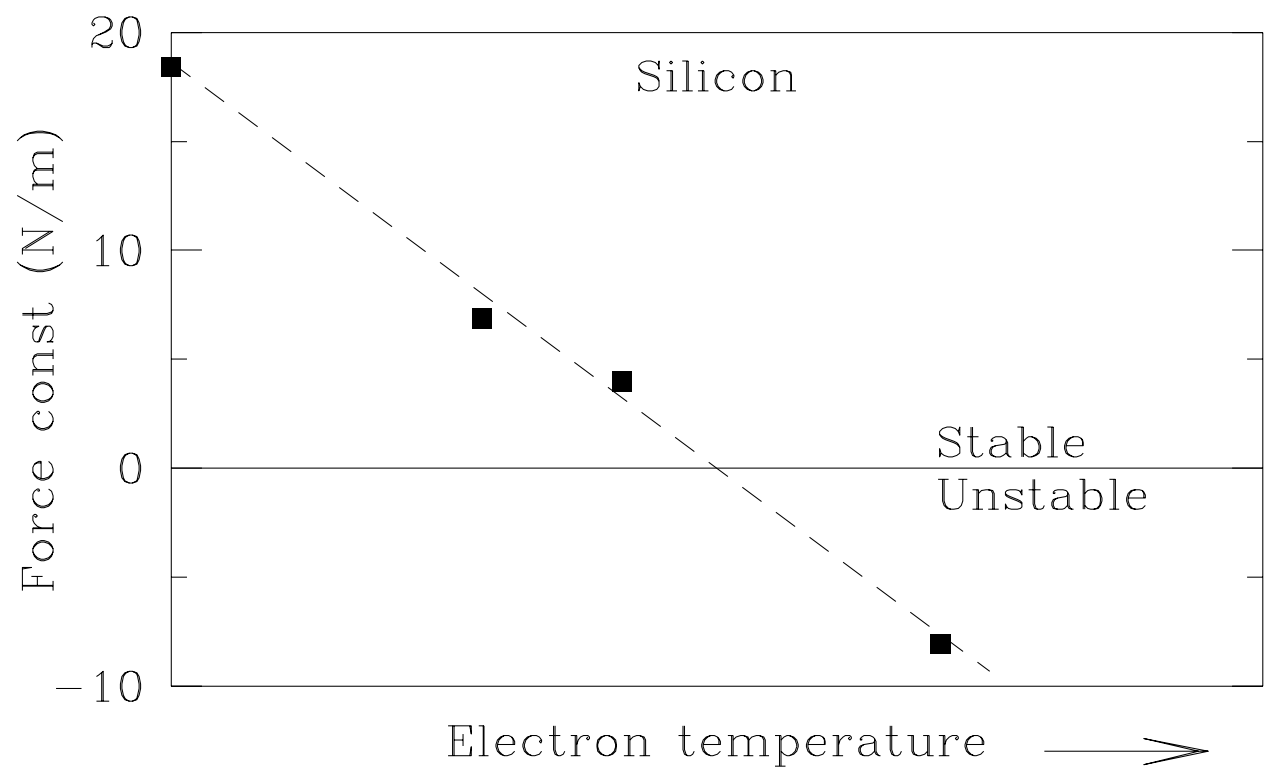

Figure 1. Calculated force constants in silicon as a function of electron temperature. With increasing temperature, the force constant becomes negative, indicating a lattice instability, as seen in ultrashort-pulse laser experiments. The squares are calculated values, and the dashed line is a guide to the eye.

Our first effort at calculating the EOS of an element at $T \sim T_{\text {Fermi }}$ (typically $10^{5} \mathrm{~K}$ ) was a 
calculation of the Hugoniot of beryllium. These calculations were performed without the use of a pseudopotential, so thermal excitation of the 1 s core electrons was automatically included. We did not perform molecular dynamics simulations, but instead calculated the free energy of a beryllium crystal with hot electrons and cold nuclei, and used an ideal gas model to calculate the contribution of ion motion to the EOS. We found that the neglect of ion motion resulted in poor agreement with existing theories, as shown in Figure 2.

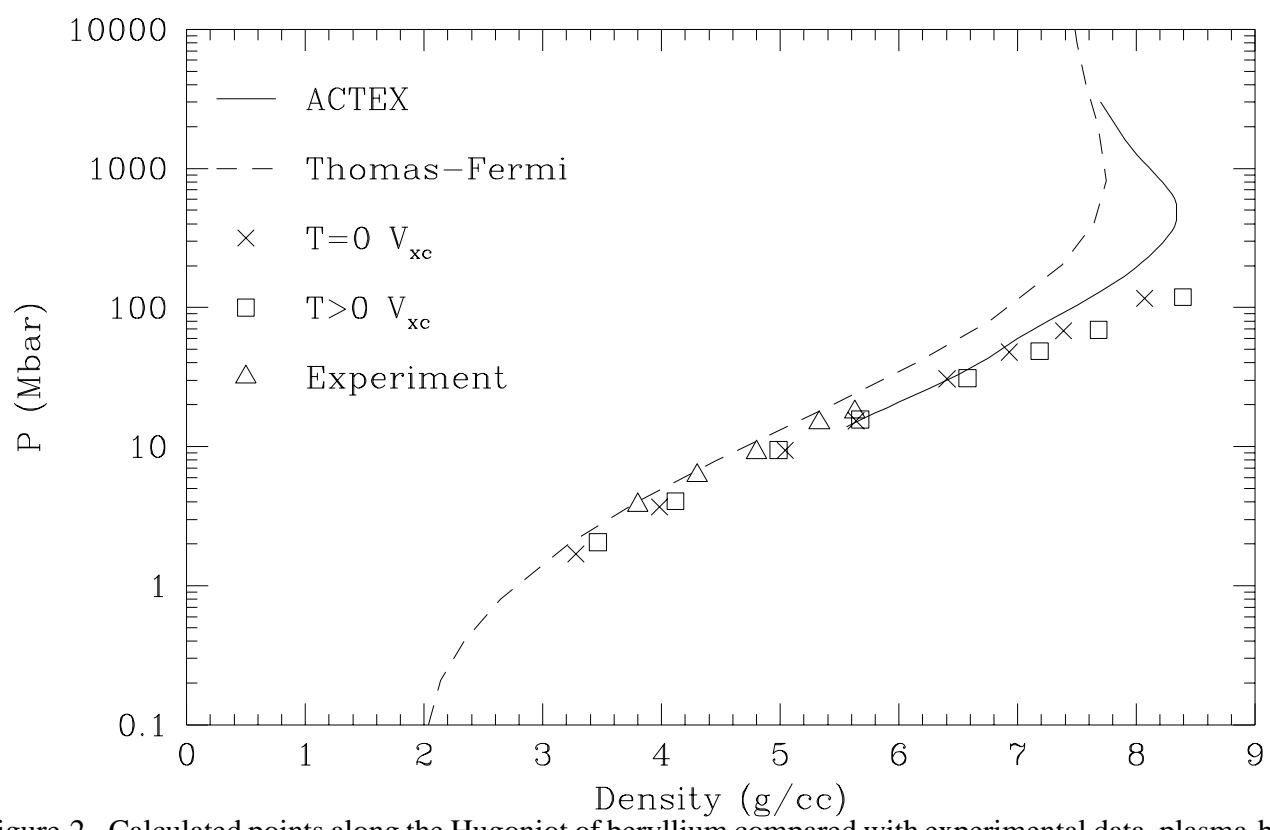

Figure 2. Calculated points along the Hugoniot of beryllium compared with experimental data, plasma-based theory (ACTEX), and simple Thomas-Fermi theory. The inclusion of a finite-temperature model for the exchange-correlation potential $V_{x c}$ moves the Hugoniot to higher density at a given pressure.

We then decided to include the effects of ion disorder on the EOS by performing molecular dynamics (MD) simulations at high temperatures. For all but the lightest elements, thermal excitation of core electrons must be included to obtain reliable results; however, including all of the core electrons in MD is computationally infeasible. We have developed a new pseudopotential formalism, derived from photoemission calculations, which we have adapted to treat atom-electron scattering at extreme temperatures. Our results, as described in [3], indicate that the calculations should be reliable for temperatures up to and exceeding one million Kelvin. This new formalism was employed to calculate the shock Hugoniot of aluminum. At the highest temperatures, we discovered that the molecular dynamics simulations can be performed with very small simulation cells, encompassing just four atoms. This is sufficient to describe the ion-ion pair correlation function out to the average nearest neighbor separation, permitting calculations up to $3 \times 10^{6} \mathrm{~K}$. As described in our paper [3], the results are in excellent agreement with experimental data and also with the previous lowtemperature calculations, and approach the high-temperature plasma-based theory at high density and temperature.

We also pursued a second line of inquiry focused on the effects of including a finite temperature quantum-mechanical description of the electron gas (as modeled by the exchange and correlation potential $V_{x c}$. We found that the predicted density of extremely strongly shocked beryllium(see Fig. 2) and aluminum [3] (at $T \geq T_{\text {Fermi }}$ ) changed by several percent when a finite-T model was used for $V_{x c}$, indicating that inclusion of finite-T effects is needed for accurate results at $T \geq T_{\text {Fermi }}$. 

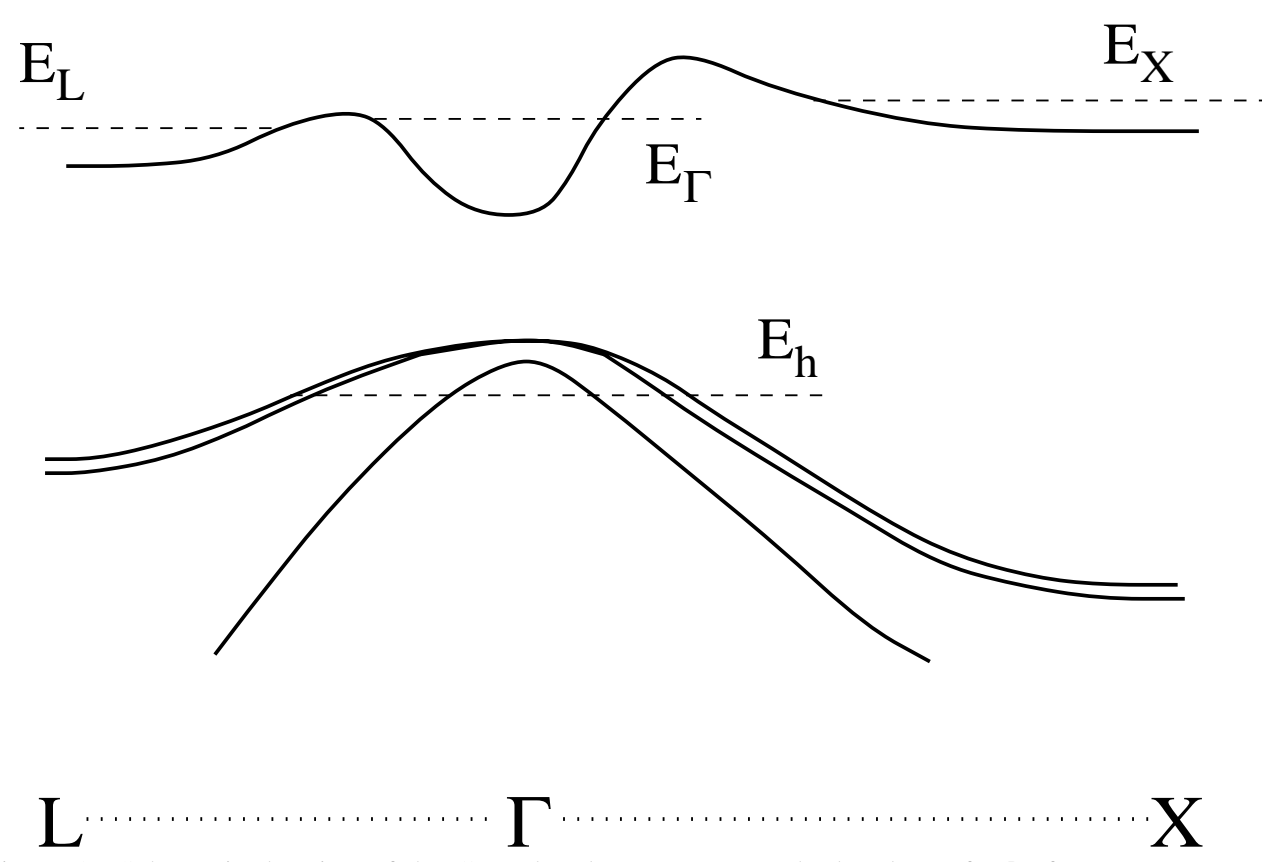

Figure 3. Schematic drawing of the GaAs band structure near the band gap for $\mathbf{k}$ from $\mathrm{L} \rightarrow \Gamma \rightarrow X$. The dashed lines indicate quasi-Fermi levels for electrons (conduction band) and holes (valence band).

We also found that for $T<<T_{\text {Fermi }}$, finite-T effects were insignificant, as we expected.

Our study of the optical properties of warm dense matter proceeded along two fronts: calculation of visible and near-UV absorption in optically pumped GaAs, and the calculation of excited state (including optical) properties of solid-density Al near the Fermi temperature. In both of these studies, we deepened our physical insight while developing the computational machinery needed to address the problem in a more general context.

Our calculations of absorption in laser-excited GaAs, published in [4], were meant to address the experiments of Mazur [5]. In these experiments, a $70 \mathrm{fs}$ pump pulse containing $1.9 \mathrm{eV}$ photons excites a GaAs crystal. A few hundred fs later, the reflectivity is probed from 1.5 to $4.0 \mathrm{eV}$ (in the vicinity of the so-called $\mathrm{E}_{1}$ peak at $3.0 \mathrm{eV}$ ). The pump fluence is high enough so as to excite in excess of $10^{21}$ carriers $/ \mathrm{cm}^{3}$ (see Figs. 3,4). At these short pump-probe delay times, the ions are thought to be close to their equilibrium lattice positions. A fundamental result of these measurements is the reduction in $\mathrm{E}_{1}$ peak height following the excitation. Our calculations using the Bethe-Salpeter equation with a screened electron-hole interaction reproduce these features, at least qualitatively (see Fig. 4). We have shown that the peak reduction results from a combination of Pauli blocking (altered probability of interband excitation) and increased screening of the electron-hole attraction (excitonic effect). However, it is possible that the excited charge responds too slowly to contribute to the screening. Unable to go beyond the static screening approximation to the Bethe-Salpeter equation (in an ab initio calculation) we posited a "quasi-static" screening approach, wherein the excited carriers are assumed to respond too slowly to contribute to the screening. The results are similar to those of Fig. 4, but with slightly larger intensity in the peak at $3 \mathrm{eV}$ for each density. The true results are likely to lie somewhere between those of the static and quasi-static cases.

The $\mathrm{Al}$ calculations are being performed to address thin foil measurements in $\mathrm{V}$ division at LLNL. Here we simulate the $\mathrm{Al}$ fluid at solid densities and temperatures of several $\mathrm{eV}$ using our quantum molecular dynamics scheme described in [3]. Electron and ion temperatures are independently var- 


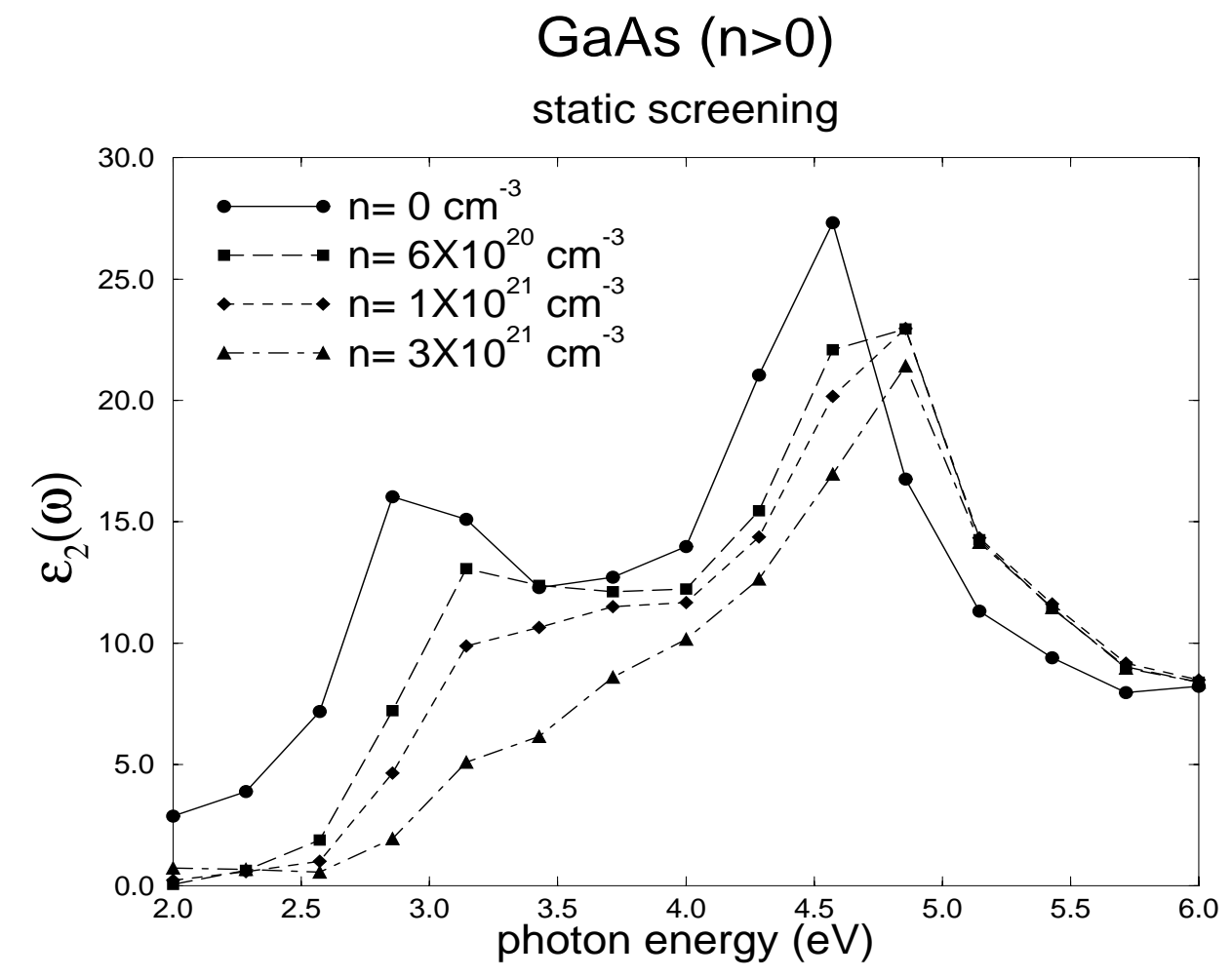

Figure 4. $\epsilon_{2}(\omega)$ vs photon energy $\omega$ for four occupation number distributions (each characterized by excited carrier density $n$ ) in the static screening approximation. As the excited carrier density $n$ increases, the $E_{1}$ peak in the absorption is strongly screened (see text).

ied. Excitonic effects are ignored in this study, due to the strong screening in (even un-pumped) Al. We find that the frequency-dependent conductivity resembles that of the Drude model for the $T_{\text {electron }}=T_{\text {ion }}=$ several $\mathrm{eV}$ cases, even at solid densities. However, the spectral broadening we have used is entirely phenomenological. The true broadening may result from the strong electronelectron scattering expected when $T \sim T_{\text {Fermi }}$. In order to address this problem, we have collaborated with the Louie group at UC Berkeley to compute the energy-dependent lifetime $\tau(E)$ of an electron in hot dense $\mathrm{Al}$ using the $\mathrm{GW}$ approximation. Results suggest that $1 / \tau\left(E=E_{\text {fermi }}\right)$ is proportional to $T^{2}$, as suggested long ago by Landau. In addition, we have learned that the amount of spectral weight in the quasiparticle peak in the electron spectral function is a very weak function of $T$ (at least within the GW approximation), suggesting that the quasiparticle concept is still meaningful at these conditions, even if the lifetime is short. Calculations of the AC conductivity for the crystalline $\left(T_{\mathrm{ion}}=0\right)$ case are currently underway. These may be the most relevant for comparing to the $\mathrm{V}$ division experiment, for their pump-probe delay time is under a ps, and the thickness of their foils is only 100 Angstroms. 


\section{SUMMARY AND FUTURE WORK}

We have developed a method for calculating the equation of state for simple materials at temperatures ranging from zero Kelvin to in excess of $10^{6} \mathrm{~K}$. Crucial elements of this method include (1) the use of molecular dynamics to account for the motion of atoms in the fluid, (2) pseudopotentials incorporating excited core states to accurately reflect atom-electron scattering at high temperature, and (3) a finite- temperature treatment of the quantum-mechanical properties of the electron gas. This method has been successfully applied to the EOS of aluminum. We have also extended zerotemperature theory for the optical properties of solids to handle the case where the electrons in the solid are in excited states, either due to elevated temperature or laser-driven excitation. We found that it was necessary to include both (1) the screening of the electron-hole interaction and (2) the modified probability of interband transitions for accurate results. Future extensions of this work will focus on the optical properties of disordered materials (e.g., hot fluids) and on the EOS of higher $Z$ elements containing localized orbitals (e.g., $d$ and $f$ orbitals).

\section{ACKNOWLEDGMENTS}

This work was performed under the auspices of the U.S. Department of Energy by the University of California Lawrence Livermore National Laboratory under Contract No. W-7405-Eng-48.

This research is funded by the Laboratory Directed Research and Development (LDRD) Program at Lawrence Livermore National Laboratory (LLNL). The LDRD Program is mandated by Congress to fund director-initiated, long-term research and development (R\&D) projects in support of the DOE and national laboratories mission areas. The Director's Office LDRD Program at LLNL funds creative and innovative R\&D to ensure the scientific vitality of the Laboratory in mission-related scientific disciplines.

\section{REFERENCES}

[1] M. C. Payne et al., Rev. Mod. Phys. 64, 1045 (1992).

[2] P. Stampfli and K. H. Bennemann, Phys. Rev. B 49, 7299 (1994) and references therein.

[3] M. P. Surh, T. W. Barbee III, and L. H. Yang, UCRL-JC-140608, submitted to Physical Review Letters, 2000.

[4] L. X. Benedict, UCRL-JC-140337, Phys. Rev. B 63, 075202 (2001).

[5] L. Huang, J. P. Callan, E. N. Glezer, and E. Mazur, Phys. Rev. Lett. 80, 185 (1998); J. P. Callan, A. M. T. Kim, L. Huang, and E. Mazur, Chem. Phys. 251, 167 (2000). 